\title{
BMJ Open Impact of team-based community healthcare on preventable hospitalisation: a population-based cohort study in Taiwan
}

Chyi-Feng Jeff Jan (D) , ${ }^{1,2}$ Che-Jui Jerry Chang, ${ }^{1}$ Shinn-Jang Hwang, ${ }^{3,4,5}$ Tzeng-Ji Chen, ${ }^{3,4}$ Hsiao-Yu Yang (1) ,6 Yu-Chun Chen, ${ }^{3,4}$ Cheng-Kuo Huang, 5,7 Tai-Yuan Chiu (D) 1,2,7

To cite: Jan C-FJ, Chang C$\mathrm{JJ}$, Hwang S-J, et al. Impact of team-based community healthcare on preventable hospitalisation: a population-based cohort study in Taiwan. BMJ Open 2021;11:e039986. doi:10.1136/ bmjopen-2020-039986

- Prepublication history for this paper is available online. To view these files, please visit the journal online (http://dx.doi. org/10.1136/bmjopen-2020039986).

Received 01 May 2020 Revised 09 December 2020 Accepted 16 December 2020

Check for updates

(C) Author(s) (or their employer(s)) 2021. Re-use permitted under CC BY-NC. No commercial re-use. See rights and permissions. Published by BMJ.

For numbered affiliations see end of article.

Correspondence to Professor Tai-Yuan Chiu; tychiu@ntuh.gov.tw

\section{ABSTRACT}

Objectives The objective of this study was to explore the impact of Taiwan's Family Practice Integrated Care Project (FPICP) on hospitalisation.

Design A population-based cohort study compared the hospitalisation rates for ambulatory care sensitive conditions (ACSCS) among FPICP participating and nonparticipating patients during 2011-2015.

Setting The study accessed the FPICP reimbursement database of Taiwan's National Health Insurance (NHI) administration containing all $\mathrm{NHI}$ administration-selected patients for FPICP enrolment.

Participants The NHI administration-selected candidates from 2011 to 2015 became FPICP participants if their primary care physicians joined the project, otherwise they became non-participants.

Interventions The intervention of interest was enrolment in the FPICP or not. The follow-up time interval for calculating the rate of hospitalisation was the year in which the patient was selected for FPICP enrolment or not.

Primary outcome measures The study's primary outcome measures were hospitalisation rates for ACSC, including asthma/chronic obstructive pulmonary disease (COPD), diabetes or its complications and heart failure.

Logistic regression was used to calculate the ORs concerning the influence of FPICP participation on the rate of hospitalisation for ACSC.

Results The enrolled population for data analysis was between 3.94 and 5.34 million from 2011 to 2015.

Compared to non-participants, FPICP participants had lower hospitalisation for COPD/asthma (28.6\% ${ }_{0}-35.9 \%$ o vs $37.9 \% 0-42.3 \%$ ) and for diabetes or its complications (10.8\%o-14.9\%o vs $12.7 \%$ - $18.1 \%$ ) but not for congestive heart failure. After adjusting for age, sex and level of comorbidities by logistic regression, participation in the FPICP was associated with lower hospitalisation for COPD/asthma (OR 0.91, 95\% Cl 0.87 to 0.94 in 2015) and for diabetes or its complications (OR $0.87,95 \% \mathrm{Cl} 0.83$ to 0.92 in 2015).

Conclusion Participation in the FPICP is an independent protective factor for preventable ACSC hospitalisation. Team-based community healthcare programs such as the FPICP can strengthen primary healthcare capacity.
Strengths and limitations of this study

- This study is the largest, population-based cohort study focused on the impact of Family Practice Integrated Care Project (FPICP).

- The intervention and control groups have high comparability as the same eligible criteria without patient selection by physicians.

- The database does not collect information about patients' lifestyle factors, which may have affected clinical outcomes.

- The study did not perform propensity score matching due to limited computing power regarding the big data.

- This observational study could only explore the association between FPICP and reduced hospitalisations, rather than causality.

\section{INTRODUCTION}

Taiwan's National Health Insurance (NHI) programme is renowned for its costeffectiveness and accessibility and serves 23.8 million people with a $99.6 \%$ coverage as a high-performing single-payer health insurance system. ${ }^{12}$ Nevertheless, Taiwan also has to face a serious challenge to its financial sustainability due to an ageing population, an insufficient insurance premium rate, as well as fragmented and less patient-centred integrated care as a result of fee-for-servicebased payments. To maintain quality care and reduce wasting of resources, Taiwan's government has been taking action with interventions and policies aimed to reinforce the healthcare capacity of primary care physicians and to re-emphasise general medical training. One major intervention is the establishment of the Family Practice Integrated Care Project (FPICP). ${ }^{3}$

In brief, the FPICP is a modified pay-forperformance $(\mathrm{P} 4 \mathrm{P})$ programme that affects 
$10 \%$ of all NHI beneficiaries. Featuring team-based care provided by primary care clinics with integration with community hospitals, the FPICP was started as a pilot project in 2003 and was reformed in 2010 as a regular government healthcare programme. The community healthcare group (CHCG), a team of 5-10 primary care physicians in a single community working in cooperation with a local hospital, forms the core healthcare unit of the FPICP. The target population of the FPICP are patients with multiple chronic diseases, frequent users of outpatient care and the elderly aged over 65 years. Taiwan's NHI administration selects those incurring higher medical costs among target patients on a yearly basis to compile a list of FPICP candidates. There is no 'cherrypicking phenomenon' because the FPICP requires the member physicians to include all administrationassigned patients of the NHI. Primary care physicians of the FPICP deliver integrated healthcare services through collaboration within the CHCG, focusing especially on preventive care, providing continuous care with 24-hour telephone hotline consultation with hospital doctors and a bidirectional mutual referral network among the primary care clinics and local hospitals. If an FPICP participant is hospitalised, the primary care physician can visit the patient in the hospital and participate in the ward round, facilitating further referral back to the primary care clinic. Until the end of 2015, approximately $25 \%$ of primary care physicians and $30 \%$ of community clinics joined the FPICP to serve the participating patients in 426 CHCGs. ${ }^{4}$

Integrated healthcare services provided by primary care physicians of the FPICP are incentivised in addition to a regular fee-for-service payment scheme. On average, each physician is responsible for $550-750$ participating FPICP patients. The programme allocates 250 points to member physicians ( 1 point $=0.9$ New Taiwan dollar or US $\$ 0.03$, with floating value per point under the global budget scheme) as a case management fee per participant per year, along with a 550 points bonus if the performance of their CHCG reaches a specified quality indicator goal (online supplemental appendix 1). The cost of FPICP is relatively low compared with its coverage rate. It requires a share of just $0.2 \%$ (US $\$ 40$ million) of Taiwan's US $\$ 20$ billion NHI annual budget. ${ }^{5}$

The impact of the FPICP since its reformation in 2010 has yet to be fully ascertained. Therefore, we aimed to quantify the progress of the FPICP towards the NHI administration's goal of fortifying primary healthcare and reducing wastage of medical resources. One indicator of choice was the hospitalisation rate for ambulatory care sensitive conditions (ACSCs), which are considered manageable by primary care physicians and as such hospital stays from ACSC can be considered preventable. $^{6}$ We hypothesised that patients participating in the FPICP would have a lower rate of hospitalisation for ACSC compared with non-participating patients.

\section{METHODS}

We conducted a population-based cohort study comparing hospitalisation rates for ACSC among FPICP participating and non-participating patients in Taiwan. The study was in compliance with the Strengthening the Reporting of Observational Studies in Epidemiology checklist of essential items (version 4) for cohort studies. ${ }^{7}$

\section{Setting}

The study used data from the FPICP database, the reimbursement database of Taiwan's NHI administration containing all NHI administration-selected patients for FPICP enrolment. The data of patients registered in FPICP in fiscal years 2011-2015 were extracted, and the follow-up time interval to calculate the rate of hospitalisation was the 1 year in which the patient was selected for FPICP enrolment.

The consulted database has a data structure and format similar to the main NHI administration reimbursement database and the National Health Insurance Research Database, on which $99.6 \%$ of Taiwan's population are enrolled. ${ }^{8}$ The database also contained comprehensive drug prescription files and original claim data. In addition to the main NHI administration reimbursement database, the database of the FPICP included four other components: a dataset on the original FPICP candidates, a dataset on the final FPICP participants, a dataset on CHCG profiles and a dataset on quality assessments of the CHCG. The database of the FPICP, with the help of these extra datasets, enables research specific to the family physician system in Taiwan and was first used in a recent publication by the authors. ${ }^{3}$

\section{Target population}

The study's target population was patients in primary care clinics eligible for FPICP inclusion in the fiscal year 2011-2015 and aged above 5years. The original version of the ACSC in the pan-Canadian primary Healthcare indicators targeted patients aged 5-75 years for the calculation of hospitalisation rates. ${ }^{9}$ However, because the FPICP focused on the elderly and patients aged over 80 accounted for $6 \%-9 \%$ of all FPICP participants, we did not apply an upper age limit for our target population.

\section{Variables}

When defining ACSC in this study, we referred to the standards set by the Canadian Institute for Health Information and modified them according to primary healthcare practice routines in Taiwan. ${ }^{6}{ }^{10}$ The outcome measures were the rates of hospitalisation for ACSC including asthma/chronic obstructive pulmonary disease (COPD), diabetes or its complications and heart failure. Rate of hospitalisation due to ACSC was calculated as the number of patients hospitalised with a main discharge diagnosis of ACSC per 1000 of the outpatient population with ACSC. Specifically, the numerator is hospitalisation with a main diagnosis of one of the conditions below: 


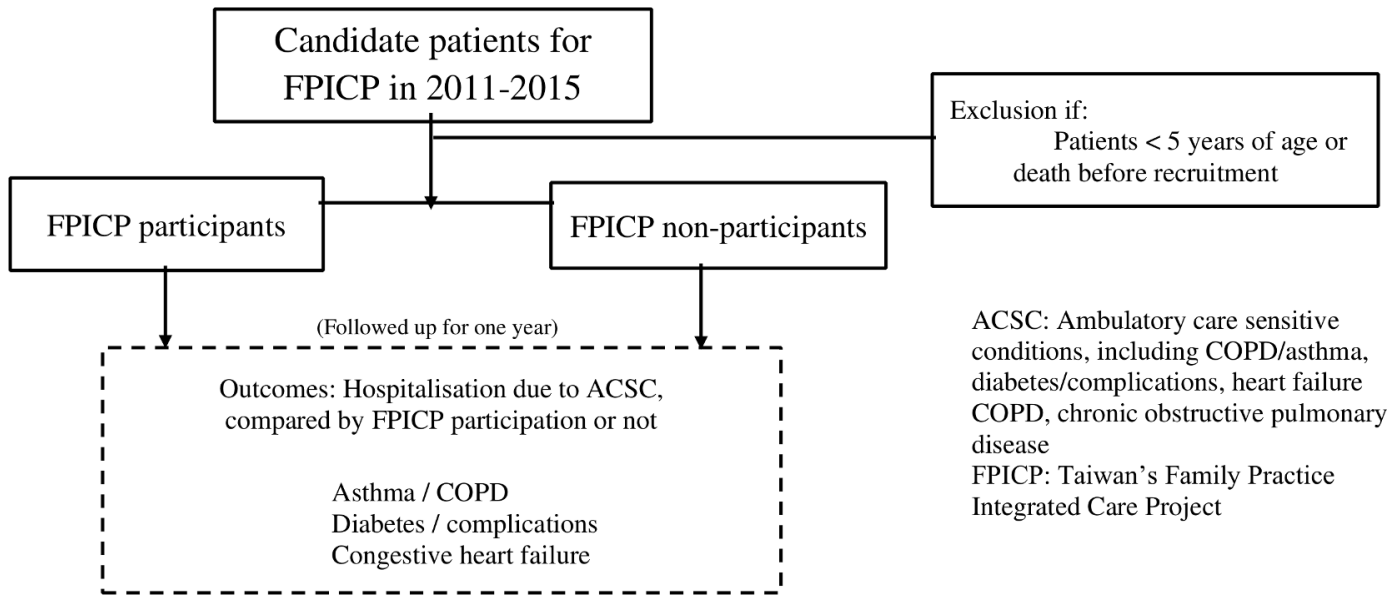

Figure 1 Flowchart of data collection. ACSC, Ambulatory care sensitive conditions, including COPD/asthma, diabetes/ complication, heart failure; COPD, chronic obstructive pulmonary disease; FPICP: Taiwan's family practive intergrated care project.

1. COPD/asthma: ICD-9-CM codes that begin with 490496 ; 480-488, and with a secondary diagnosis 490-496; or ICD-10-CM codes that begin with J10.0-J18, or J20$\mathrm{J} 22$, and with a secondary diagnosis J40-J47.

2. Diabetes and its complications: ICD-9-CM codes that begin with 250; or ICD-10-CM codes that begin with E10, E11, E13.

3. Heart failure: ICD-9-CM codes that begin with 428 or 518.4; or ICD-10-CM codes that begin with I50 or J81.

The denominator is the population with an outpatient diagnosis of ACSC in the previous year.

The intervention of interest was enrolment in the FPICP or not (figure 1). These NHI Administrationselected candidates became FPICP participants if their primary care physicians joined the project and enrolled all assigned patients as participants. Factors regarded as potential confounders included age, sex, monthly income, region of residence and comorbidities. Monthly income and region of residence were based on the Registry for Beneficiaries dataset obtained on a study subject's enrolment in the NHI programme. Comorbidities were assessed using the Charlson Comorbidity Index (CCI) ${ }^{11} 12$ We defined the diagnosis of a comorbidity as receiving the same diagnosis no less than twice in the previous year based on the ICD-9-CM and ICD-10-CM codes as indicated by physicians' claims data. The ICD codes used in this study are described in online supplemental appendix 2. The technical part of CCI calculation was based on the open-sourced SAS scripts published by Healthcare Delivery Research at the National Cancer Institute of the USA. ${ }^{13}$

We converted the quantitative variables into categorical ones as follows: older adults were defined as participants aged 65 years or older, which is consistent with the WHO's definition ${ }^{14}$; monthly income was categorised by tertiles; codes of residential region were transformed into three levels of urbanisation according to Taiwan National Health Research Institue (NHRI) publications, with level 1 referring to 'most urbanised' and level 3 'least urbanised' communities; increased comorbidity score was defined as a CCI of 3 or greater, which was adopted or suggested by previous studies. ${ }^{115}$

\section{Statistical analysis}

Values were presented either as percentages or as arithmetic means with SD in descriptive analyses. Logistic regression was used to calculate the ORs for the influence of FPICP participation on the rate of hospitalisation for ACSC. Age (in dichotomised categories by 65 years old), gender and level of comorbidities were included as independent variables in the model. A two-tailed $p$ value of 0.05 was considered statistically significant, and $95 \%$ CIs were also calculated. Propensity score matching for FPICP participating and non-participating patients was not applied due to the large number of observations (1-2 million participants per year) and limited computing power. All statistical analyses were conducted using SAS software (V.9.4SAS Institute).

\section{Patient and public involvement}

We did not directly include patient and public involvement in this study, but the database used in the study was developed with patient and public involvement and is updated by a committee that includes patient representatives from the NHI Administration, Ministry of Health and Welfare, Taiwan.

\section{RESULTS}

After excluding children under the age of five and patients who had dropped out from the NHI programme before recruitment (deceased or moved away), the study population, including participants and non-participants, was 3.94 million in 2011 and 5.34 million in 2015 (online supplemental appendix 3). Among them, the population of FPICP participants was 2316114 (43.4\%) for 2015.

Table 1 summarises the demographic characteristics of the study participants in 2015. For FPICP participants, 
Table 1 Characteristics of study base at enrolment in 2015

\begin{tabular}{lll} 
& FPICP & Non-FPICP \\
\hline $\begin{array}{l}\text { Number of } \\
\text { observation }\end{array}$ & 2316114 & 3021263
\end{tabular}

Sex

\begin{tabular}{|c|c|c|}
\hline Female & 1241437 (53.6\%) & $1613354(53.4 \%)$ \\
\hline Male & 1074677 (46.4\%) & 1407908 (46.6\%) \\
\hline \multicolumn{3}{|l|}{ Age (years)* } \\
\hline $5-10$ & 222703 (9.6\%) & 239933 (7.9\%) \\
\hline $10-20$ & 252397 (10.9\%) & $303073(10.0 \%)$ \\
\hline 20-30 & $155892(6.7 \%)$ & 161008 (5.3\%) \\
\hline $30-40$ & $259820(11.2 \%)$ & 287288 (9.5\%) \\
\hline $40-50$ & 291989 (12.6\%) & 344114 (11.4\%) \\
\hline $50-60$ & 381070 (16.5\%) & $486180(16.1 \%)$ \\
\hline $60-70$ & 351376 (15.2\%) & 524064 (17.3\%) \\
\hline $70-80$ & 249923 (10.8\%) & 423040 (14.0\%) \\
\hline $80-90$ & $131147(5.7 \%)$ & $217834(7.2 \%)$ \\
\hline over 90 & $19797(0.8 \%)$ & $34728(1.3 \%)$ \\
\hline \multicolumn{3}{|l|}{ Monthly income†‡ } \\
\hline Level 1 (high) & 826853 (35.7\%) & $1078591(35.7 \%)$ \\
\hline Level 2 (medium) & 817588 (35.3\%) & $1102761(36.5 \%)$ \\
\hline Level 3 (low) & $671673(29.0 \%)$ & $839910(27.8 \%)$ \\
\hline \multicolumn{3}{|l|}{ Urbanisation‡ } \\
\hline Level 1 (high) & 528074 (22.8\%) & $797613(26.4 \%)$ \\
\hline Level 2 (medium) & 1100154 (47.5\%) & $1326334(43.9 \%)$ \\
\hline Level 3 (low) & 687886 (29.7\%) & 897315 (29.7\%) \\
\hline
\end{tabular}

*Age at enrolment.

tCounted in New Taiwan dollar (NTD).

‡Categorised by tertiles.

FPICP, Taiwan's Family Practice Integrated Care Project;

$53.6 \%$ of them were female, $17.3 \%$ were aged over 70 and $20.5 \%$ were aged under 20 . As differences were small between FPICP participants and non-participants in terms of monthly income $(29.0 \%$ vs $27.8 \%$ in the low-income category) and urbanisation level of their residential area (29.7\% vs $29.7 \%$ in the low-urbanisation category), we did not include monthly income and the urbanisation as independent variables in the logistic regression analysis.

Table 2 shows that the outpatient population of patients with ACSC (COPD/asthma, diabetes or heart failure) was 366047 among FPICP participants and 481600 among non-participants in 2015. FPICP participants had a smaller proportion of patients with CCI $>2$ (by $1.4 \%$ ), reduced medical costs per year for outpatient department (OPD)/clinic visits (by $9.0 \%-15.2 \%$ ), for ER visits (by $11.4 \%-14.5 \%$ ) and for hospitalisation (by $17.5 \%-$ 19.3\%). (full comparison from 2011 to 2015 in online supplemental appendix 4).

Figure 2 shows the rate of hospitalisation for selected ACSCs from 2011 to 2015. Compared with nonparticipants, FPICP participants had lower hospitalisation for COPD/asthma (37.9\%o-42.3\%o vs $28.6 \%$ o-35.9\%o) and for diabetes or its complications $(12.7 \%$ o- $18.1 \%$ vs
Table 2 Comorbidities and utilisation of medical resource among patients with ACSC, by FPICP participation (2015)

\begin{tabular}{lll}
\hline & FPICP & Non-FPICP \\
\hline $\begin{array}{l}\text { Number of observation } \\
\text { CCl }\end{array}$ & 366047 & 481600 \\
\hline High (>2) & $52656(14.4)$ & $76019(15.8)$ \\
\hline Low (0-2) & $313391(85.6)$ & $405581(84.2)$ \\
\hline $\begin{array}{l}\text { Clinic/outpatient care } \\
\text { Number of visits/year }\end{array}$ & $12.6(12.1)$ & $14.0(13.3)$ \\
\hline $\begin{array}{l}\text { Medical cost/year } \\
\text { (point) }\end{array}$ & $9458(67,589)$ & $10655(34,250)$ \\
\hline $\begin{array}{l}\text { Emergency care } \\
\text { Number of visits/year }\end{array}$ & $0.43(1.49)$ & $0.45(1.44)$ \\
\hline $\begin{array}{l}\text { Medical cost/year (point) } \\
\text { Inpatient care }\end{array}$ & $1365(5670)$ & $1541(7400)$ \\
\hline $\begin{array}{l}\text { Number of visits/year } \\
\text { Medical cost/year (point) }\end{array}$ & $0.30(0.77)$ & $0.35(0.85)$ \\
\hline $\begin{array}{l}\text { Hospitalisation rate } \\
\text { Length of stay (day) }\end{array}$ & $16.3 \%$ & $22733(96611)$ \\
\hline
\end{tabular}

$\mathrm{SD}$ or percentage is shown in parentheses.

*Floating point value (1 point NT\$0.9) under global budget scheme since 2001.

ACSC, ambulatory care sensitive conditions; CCl, Charlson Comorbidity Index; FPICP, Taiwan's Family Practice Integrated Care Project.

$10.8 \%$ - $14.9 \% 0)(\mathrm{p}<0.05)$. The reduced hospitalisation rate for heart failure was also noted, but there was no statistical significance $(49.6 \%$ - $54.1 \%$ vs $43.9 \%$ o-50.6\%o).

After adjusting for age, sex and level of comorbidities by conditional logistic regression, participation in the FPICP was associated with lower hospitalisation for COPD/ asthma (OR $0.91,95 \%$ CI 0.87 to 0.94 ) and for diabetes or its complications (OR $0.87,95 \%$ CI 0.83 to 0.92 ) but not for heart failure (OR $0.97,95 \%$ CI 0.88 to 1.07 ) (table 3 ).

\section{DISCUSSIONS}

Main findings

The FPICP has been the most important reform programme for primary healthcare in Taiwan since 2010, and to our knowledge, this study is the first to directly use real-world data from the FPICP to verify the effectiveness of the programme. Moreover, the reason for reporting the outcome of ACSC is that the quality of care for these diseases can be reflected in a reduction in the use of hospital resources if well controlled at primary healthcare clinics. We found that participants in the FPICP presented a lower hospitalisation rate regarding ACSC, including asthma/COPD and diabetes or its complications. After adjusting for variables such as age, sex and comorbidities, participation in the FPICP remains an independent protective factor for preventable hospitalisation. This major finding sheds light on the team-based primary healthcare model such as FPICP strengthened primary 


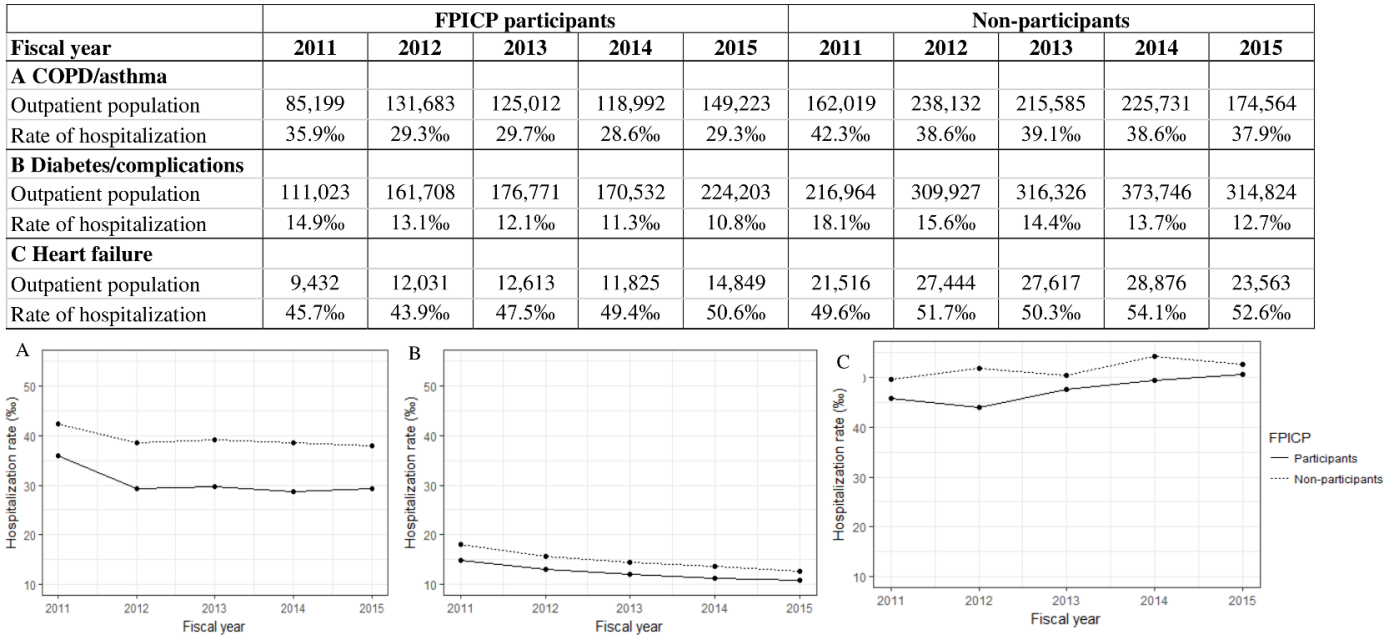

Figure 2 Rate of hospitalisations for ACSC. ACSC, Amubulatory care sensitive conditions; COPD, Chronic obstructive pulmonary disease; FPICP, Taiwan's Family Practice Integrated Care Project. Source: Author's analysis of data from the National Health Insurance Administration, Taiwan.

healthcare capacity and improved quality of community healthcare. ${ }^{16}$

Other than the above findings, FPICP participants were also found to have lower medical costs as outpatients, through emergencies and through hospitalisation, compared with the non-participants. The difference in hospitalisation costs is particularly significant $(17.5 \%-$ $19.3 \%)$, followed by emergency costs $(11.4 \%-14.5 \%)$ and outpatient expenses (by $17.5 \%-19.3 \%$ ).

Our study supported the evidence that by engaging in data-driven, continuous quality improvement, teambased care can offer higher accessibility to care, as well as more effective and efficient delivery by providing care coordination. ${ }^{17-20}$ In Canada, Carter et al found moderate quality evidence that team-based models of care led to reductions in emergency department use, but the evidence was mixed for hospital admissions. ${ }^{21}$ McAlister et al also demonstrated that care within a primary care network was associated with fewer emergency department visits and fewer hospital days. ${ }^{22}$

Regarding disease-specific team-based care examples, a meta-analysis done by Carter et al demonstrated that

\begin{tabular}{lll}
\hline $\begin{array}{l}\text { Table } 3 \\
2015\end{array}$ & FPICP and reduced hospitalisation for ACSC in \\
\hline $\begin{array}{l}\text { Hospitalisation for } \\
\text { ACSC }\end{array}$ & $\begin{array}{l}\text { Absolute rate } \\
\text { reduction, \%o } \\
(95 \% \text { Cl) }\end{array}$ & OR $(95 \%$ Cl) \\
\hline COPD/asthma & $8.6(7.4$ to 9.8$)$ & $0.91(0.87$ to 0.94$)$ \\
$\begin{array}{l}\text { Diabetes and the } \\
\text { complications }\end{array}$ & $1.9(1.3$ to 2.5$)$ & $0.87(0.83$ to 0.92$)$ \\
Heart failure & $2.0(-2.5$ to 6.5$)$ & $0.97(0.88$ to 1.07$)$ \\
\hline
\end{tabular}

The ORs and $95 \% \mathrm{Cl}$ (in parentheses) were estimated using conditional logistic regressions. Other independent variables for adjusted ORs include age, gender and comorbidities.

.ACSC, ambulatory care sensitive conditions; COPD, chronic obstructive pulmonary disease; FPICP, Taiwan's Family Practice Integrated Care Project. team-based care was associated with improved blood pressure control. ${ }^{23}$ Proper training, the use of an electronic clinical reminder system and the enhanced engagement of registered nurses can help to improve completion rates of asthma action plans in a team-based primary care setting. ${ }^{24}$ As to diabetes care, team-based care management interventions that uise nurses, medical assistant health coaches, and behavioural specialists to support diabetes patients can help primary care practices achieve value-based targets of improved health, cost and patient experience. $^{25}$ Furthermore, in patients with COPD, a team-based approach following the treatment guidelines of Global Initiative for Chronic Obstructive Lung Disease is critical to successfully implement comprehensive care. ${ }^{26}$

As to hospitalisations for heart failure, the lack of a significant difference between enrolled and unenrolled participants was observed from 2011 to 2015, except in 2012. There are several possible explanations. First, a lack of close cooperation with heart failure care teams and low-intensity transitional care may have contributed to the limited efficacy. Treatment for heart failure patients in Taiwan is usually referred to cardiologists rather than follow-up at community-based clinics because diagnostic procedures need to be done such as echocardiograms or interventional studies in Taiwan. Second, the hospitalisation rate for enrolled persons with heart failure was indeed lower than non-enrolled across 20112015 although there was no statistical difference. To be noted, the cases numbers for hear failure hospitalisation were smaller compared with diabetes or COPD cases (figure 2, online supplemental appendix 5-7). As larger the number of cases, the higher the statistical power, and more likely the difference to be significant. Moreover, referring patients to specialised outpatient heart failure clinics, staffed with trained healthcare providers who are familiar with current guidelines and available resources, has been shown to reduce hospital admissions. ${ }^{27-29}$ High- or moderate-intensity transitional care 
interventions combining home visits with follow-up telephone calls, clinic visits or both reduced readmission risk if implemented for a longer period, for example, at least 6 months. ${ }^{30}$ One Cochrane review from Takeda et al in 2019 found low quality of evidence that multidisciplinary interventions instead of clinic-based interventions may reduce the risk of readmission for heart failure. Variations in study location and time of occurrence hamper attempts to review costs and cost-effectiveness. ${ }^{31}$

What Taiwan FPICP highlights is on integrated care. Primary care physicians of FPICP regularly share case discussions with medical specialists in the hospitals, so the care for chronic diseases might be more in line with current clinical guidelines. These better medical cares are reflected in lower hospitalisation rates among patients with diabetes and COPD; however, for patients with heart failure, shared care by cardiologists in the hospitals is often needed and sometimes the patients may require planned hospitalisation to do interventions; therefore, the advantages of FPICP in primary care system are more difficult to be reflected. This may help explain why there might not have been a significant difference in hospitalisations between enrolees and non-enrolees with heart failure.

Above all, FPICP as a team-based care model encourages community clinics and hospitals to form cooperative networks to facilitate the improvement of quality care, through data-driven, continuous care and coordination.

\section{Policy implications}

The quality assessment of the FPICP involves processes of care which are primarily preventive healthcare services, such as influenza vaccinations, adult regular health examinations and nationwide cancer screenings including Pap smear, fecal immunochemical tests and oral cancer screening by inspection. ${ }^{4}$ The ACSC hospitalisation rate highlighted in this study is only approximately one-tenth of all quality assessment items for the FPICP (online supplemental appendix 1). It is worth noting that the FPICP assesses quality indicators in a group-wise manner, scoring each CHCG instead of individual physicians. The rewards such as bonus payments and options to include more patients were also granted based on the performance of the CHCG. Physicians within a CHCG work as a group to review their performance and facilitate two-way coordination with their backup hospitals through regular monthly meetings. This healthcare model may be one of the keys to improving the overall quality of care in the community.

Except for copayments or out-of-pocket expense differences, whether patients are confident of their diseases being handled in community clinics is a crucial factor in forming effective CHCGs to achieve universal health coverage. If a community clinic is not capable of providing care for nearby patients who fail to seek medical help in outpatient departments of hospitals due to expense issues, the long-term consequence is likely to increase medical costs in terms of emergencies and hospitalisation. While reducing medical expenses in various ways is imperative for policymakers, patients care about the quality and accessibility of medical care. Minimising preventable hospitalisations by strengthening the ambulatory care capability of primary clinics is one of a few approaches that improves medical quality while maintaining or even reducing overall medical expenses, satisfying both patients and payers of the healthcare system. Potentially, an effective programme such as the FPICP might help enhance patients' trust in their family doctors and decrease unnecessary emergency visits or hospitalisations.

\section{Limitations}

Similar to previous database studies using physician claim data, our research, which was based on reimbursement databases, also has some limitations. ${ }^{62}$ First, we did not acquire data regarding patients' diets, physical activities, alcohol/cigarette consumption, etc., and these potential confounding factors may affect clinical outcomes. Second, we were not able to apply propensity score matching techniques due to limitations in computing power for the huge amount of data acquired, although the FPICP participating and non-participating patients were mostly assigned by NHI administration based on the same criteria. Nonetheless, we applied multiple logistic regression to adjust for potential differences in demographics and comorbidities. Third, our research only determined whether an association existed between the FPICP and the outcomes of interest, rather than causality. If an association was significant, it could be that the FPICP led to better outcomes or that physicians with better clinical ability were more likely to join the FPICP and be rewarded.

In conclusion, the FPICP is a team-based care model and a modified $\mathrm{P} 4 \mathrm{P}$ programme. It features mandatory inclusion of NHI administration-assigned patients with high medical needs in ambulatory care, and operates through a CHCG formed by local clinics, is vertically coordinated with regional hospitals. Our study adopted a population-based cohort design to validate the effectiveness of this model and found that participation in the FPICP is an independent protective factor for preventable hospitalisation. The observed trend also showed lower overall medical costs in FPICP participating patients. The experience of the FPICP may serve as a reference for policymakers in developing primary care reform programmes in order to achieve universal health coverage and improve the quality of community healthcare.

\section{Author affiliations}

${ }^{1}$ Family Medicine, National Taiwan University Hospital, Taipei, Taiwan

${ }^{2}$ Family Medicine, National Taiwan University College of Medicine, Taipei, Taiwan

${ }^{3}$ Family Medicine, National Yang-Ming Medical College, Taipei, Taiwan

${ }^{4}$ Family Medicine, Taipei Veterans General Hospital, Taipei, Taiwan

${ }^{5}$ Taiwan Association of Family Medicine, Taipei, Taiwan

${ }^{6}$ Department of Public Health and Institute of Occupational Medicine and Industrial

Hygiene, National Taiwan University College of Public Health, Taipei, Taiwan

${ }^{7}$ Taiwan Medical Association, Taipei, Taiwan 
Correction notice This article has been corrected since it first published. The provenance and peer review statement has been included.

Twitter Chyi-Feng Jeff Jan @youthDr36

Acknowledgements This study was based in part on a healthcare database provided by the National Health Insurance Administration (Taiwan). The interpretation and conclusions contained herein do not represent those of National Health Insurance Administration.

Collaborators Taiwan Association for Family Medicine.

Contributors All of the authors contributed significantly to the study. CFJ contributed to the study design review and literature review, as well as draft writing. CJC conducted the literature review and designed the study and implementation, as well as draft writing. YCC and TJC contributed to the study design and provided statistical support. SJH, HYY and CKH contributed to quality assurance and control. TYC contributed to the study design review and the data ascertainment, as well as finalised draft writing.

Funding This work was supported by the Taiwan Association of Family Medicine (TAFM 2019-2020) and Ministry of Science and Technology in 2018-2019 (MOST 107-2314-B-002-229).

Competing interests None declared.

Patient and public involvement Patients and/or the public were involved in the design, or conduct, or reporting, or dissemination plans of this research. Refer to the Methods section for further details.

Patient consent for publication Not required.

Ethics approval This article does not refer to any studies conducted by the authors on human or animal subjects. This study was approved by the ethical review board of the National Taiwan University Hospital. (NTUH201711086RIND).

Provenance and peer review Not commissioned; externally peer reviewed.

Data availability statement Data may be obtained from a third party and are not publicly available. The data that support the findings of this study are available from National Health Insurance Administration (Taiwan), but restrictions apply to the availability of these data, which were used under license for the current study, and so are not publicly available. Data are however available from the authors upon reasonable request and with permission of National Health Insurance Administration (Taiwan).

Open access This is an open access article distributed in accordance with the Creative Commons Attribution Non Commercial (CC BY-NC 4.0) license, which permits others to distribute, remix, adapt, build upon this work non-commercially, and license their derivative works on different terms, provided the original work is properly cited, appropriate credit is given, any changes made indicated, and the use is non-commercial. See: http://creativecommons.org/licenses/by-nc/4.0/.

\section{ORCID iDs}

Chyi-Feng Jeff Jan http://orcid.org/0000-0003-2893-2187

Hsiao-Yu Yang http://orcid.org/0000-0001-5298-2462

Tai-Yuan Chiu http://orcid.org/0000-0002-8528-7981

\section{REFERENCES}

1 Cheng T-M. Reflections on the 20th anniversary of Taiwan's singlepayer National Health Insurance system. Health Aff 2015;34:502-10.

2 Lee M-C. Integrated care and training in family practice in the 21st century: Taiwan as an example. Fam Med Community Health 2016;4:57-9.

3 Jan C-F, Hwang S-J, Chang C-J, et al. Family physician system in Taiwan. J Chin Med Assoc 2020;83:117-24.

4 Jan C-F, Chiu T-Y, Chen C-Y, et al. A 10-year review of health care reform on Family Practice Integrated Care Project-Taiwan experience. Fam Pract 2018;35:352-7.

5 National Health Insurance Annual Report 2017-2018. National health insurance administration, Ministry of health and welfare (Taiwan) 2018.

6 Brown AD, Goldacre MJ, Hicks N, et al. Hospitalization for ambulatory care-sensitive conditions: a method for comparative access and quality studies using routinely collected statistics. Can J Public Health 2001;92:155-9.
7 Vandenbroucke JP, von Elm E, Altman DG, et al. Strengthening the Reporting of Observational Studies in Epidemiology (STROBE): explanation and elaboration. PLoS Med 2007;4:e297.

8 National Health Insurance Research Database, Taiwan. Available: https://nhird.nhri.org.tw/en/index.html

9 Sullivan-Taylor P, Webster G, Mukhi S. Development of electronic medical record content standards to collect pan-Canadian primary health care indicator data, 2009.

10 Caminal J, Starfield B, Sánchez E, et al. The role of primary care in preventing ambulatory care sensitive conditions. Eur J Public Health 2004;14:246-51.

11 Charlson M, Szatrowski TP, Peterson J, et al. Validation of a combined comorbidity index. J Clin Epidemiol 1994;47:1245-51.

12 Charlson ME, Pompei P, Ales KL, et al. A new method of classifying prognostic comorbidity in longitudinal studies: development and validation. J Chronic Dis 1987;40:373-83.

13 Klabunde CN, Potosky AL, Legler JM, et al. Development of a comorbidity index using physician claims data. $J$ Clin Epidemiol 2000;53:1258-67.

14 Proposed working definition of an older person in Africa for the MDS project. Available: https://www.who.int/healthinfo/survey/ ageingdefnolder/en/

15 Liu C-Y, Hung Y-T, Chuang Y-L, et al. Incorporating development stratification of Taiwan townships into sampling design of large scale health interview survey. J Health Manag 2006;4:1-22.

16 Mitchell PH, Wynia MK, Golden R, et al. Core principles \& values of effective team-based health care. NAM Perspectives 2012;2.

17 Peikes DN, Reid RJ, Day TJ, et al. Staffing patterns of primary care practices in the comprehensive primary care initiative. Ann Fam Med 2014;12:142-9.

18 Aiken LH. Achieving an interdisciplinary workforce in health care. $N$ Engl J Med 2003;348:164-6.

19 Sinsky CA, Willard-Grace R, Schutzbank AM, et al. In search of joy in practice: a report of 23 high-functioning primary care practices. Ann Fam Med 2013;11:272-8.

20 Wagner EH, Flinter M, Hsu C, et al. Effective team-based primary care: observations from innovative practices. BMC Fam Pract 2017:18:13.

21 Carter R, Riverin B, Levesque J-F, et al. The impact of primary care reform on health system performance in Canada: a systematic review. BMC Health Serv Res 2016;16:324.

22 McAlister FA, Bakal JA, Green L, et al. The effect of provider affiliation with a primary care network on emergency department visits and hospital admissions. CMAJ 2018;190:E276-84.

23 Carter BL, Rogers M, Daly J, et al. The potency of team-based care interventions for hypertension: a meta-analysis. Arch Intern Med 2009;169:1748-55.

24 Kaferle JE, Wimsatt LA. A team-based approach to providing asthma action plans. J Am Board Fam Med 2012;25:247-9.

25 Fortmann AL, Walker C, Barger K, et al. Care team integration in primary care improves one-year clinical and financial outcomes in diabetes: a case for value-based care. Popul Health Manag 2020;23:467-75.

26 Amalakuhan B, Adams SG. Improving outcomes in chronic obstructive pulmonary disease: the role of the interprofessional approach. Int J Chron Obstruct Pulmon Dis 2015;10:1225-32.

27 Cooper LB, Hernandez AF. Assessing the quality and comparative effectiveness of team-based care for heart failure: who, what, where, when, and how. Heart Fail Clin 2015;11:499-506.

28 Fonarow GC, Stevenson LW, Walden JA, et al. Impact of a comprehensive heart failure management program on hospital readmission and functional status of patients with advanced heart failure. J Am Coll Cardiol 1997;30:725-32.

29 Rich MW, Beckham V, Wittenberg C, et al. A multidisciplinary intervention to prevent the readmission of elderly patients with congestive heart failure. N Engl J Med 1995;333:1190-5.

30 Vedel I, Khanassov V. Transitional care for patients with congestive heart failure: a systematic review and meta-analysis. Ann Fam Med 2015:13:562-71.

31 Takeda A, Martin N, Taylor RS, et al. Disease management interventions for heart failure. Cochrane Database Syst Rev 2019;1:CD002752.

32 Chang C-J, Yang Y-H, Chen P-C, et al. Stomach cancer and exposure to talc powder without asbestos via Chinese herbal medicine: a population-based cohort study. Int J Environ Res Public Health 2019;16:717. 